Sub-millisecond electron density profile measurement at the JET tokamak with the fast lithium beam emission spectroscopy system

Refy, D.

AIP Publishing

2018

Refy , D , JET Contributors \& Ahlgren , T 2018 , Sub-millisecond electron density profile measurement at the JET tokamak with the fast lithium beam emission spectroscopy system . in Overview and summary reports from the 26th Fusion Energy Conference (Kyoto, Japan, pÿ17 22 October 2016) . , 043509 , Review of Scientific Instruments , no. 4 , vol. 89 , AIP Publishing, Melville, NY, Fusion Energy Conference, Kyoto , Japan , 16/04/2018 . https://doi.org/10.1063/1.498662

http://hdl.handle.net/10138/326448

https://doi.org/10.1063/1.4986621

unspecified

publishedVersion

Downloaded from Helda, University of Helsinki institutional repository.

This is an electronic reprint of the original article.

This reprint may differ from the original in pagination and typographic detail.

Please cite the original version. 


\section{Sub-millisecond electron density profile}

measurement at the JET tokamak with the fast lithium beam emission spectroscopy system

Cite as: Rev. Sci. Instrum. 89, 043509 (2018); https://doi.org/10.1063/1.4986621

Submitted: 06 June 2017 . Accepted: 23 March 2018. Published Online: 20 April 2018

(iD) D. I. Réfy, M. Brix, R. Gomes, B. Tál, (D) S. Zoletnik, D. Dunai, G. Kocsis, S. Kálvin, T. Szabolics, and JET Contributors

COLLECTIONS

EP This paper was selected as an Editor's Pick

\section{ARTICLES YOU MAY BE INTERESTED IN}

Development of a high current $60 \mathrm{keV}$ neutral lithium beam injector for beam emission spectroscopy measurements on fusion experiments

Review of Scientific Instruments 89, 013503 (2018); https://doi.org/10.1063/1.5004126

Ultrafast two-dimensional lithium beam emission spectroscopy diagnostic on the EAST tokamak

Review of Scientific Instruments 89, 063503 (2018); https://doi.org/10.1063/1.5017224

Publisher's Note: "Sub-millisecond electron density profile measurement at the JET tokamak with the fast lithium beam emission spectroscopy system" [Rev. Sci. Instrum. 89, 043509 (2018)]

Review of Scientific Instruments 89, 069902 (2018); https://doi.org/10.1063/1.5043551

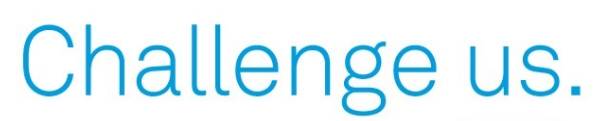

What are your needs for periodic signal detection?

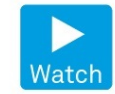

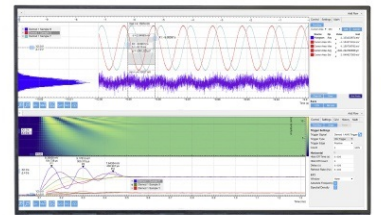

Zurich

O 


\title{
Sub-millisecond electron density profile measurement at the JET tokamak with the fast lithium beam emission spectroscopy system
}

\author{
D. I. Réfy, ${ }^{1}$ M. Brix ${ }^{2}$ R. Gomes,${ }^{3}$ B. Tál, ${ }^{1}$ S. Zoletnik, ${ }^{1}$ D. Dunai, ${ }^{1}$ G. Kocsis, ${ }^{1}$ S. Kálvin, ${ }^{1}$ \\ T. Szabolics, ${ }^{1}$ and JET Contributors ${ }^{\text {a) }}$ \\ ${ }^{1}$ Wigner Research Centre for Physics, XII Konkoly Thege Miklós út 29-33, Budapest 1121, Hungary \\ ${ }^{2}$ CCFE, Culham Science Centre, Abingdon, Oxon OX14 3DB, United Kingdom \\ ${ }^{3}$ Instituto de Plasmas e Fusão Nuclear, Instituto Superior Técnico, Universidade de Lisboa, 1049-001 Lisboa, \\ Portugal
}

(Received 6 June 2017; accepted 23 March 2018; published online 20 April 2018; publisher error corrected 23 April 2018)

\begin{abstract}
Diagnostic alkali atom (e.g., lithium) beams are routinely used to diagnose magnetically confined plasmas, namely, to measure the plasma electron density profile in the edge and the scrape off layer region. A light splitting optics system was installed into the observation system of the lithium beam emission spectroscopy diagnostic at the Joint European Torus (JET) tokamak, which allows simultaneous measurement of the beam light emission with a spectrometer and a fast avalanche photodiode (APD) camera. The spectrometer measurement allows density profile reconstruction with $\sim 10 \mathrm{~ms}$ time resolution, absolute position calculation from the Doppler shift, spectral background subtraction as well as relative intensity calibration of the channels for each discharge. The APD system is capable of measuring light intensities on the microsecond time scale. However $\sim 100 \mu$ s integration is needed to have an acceptable signal to noise ratio due to moderate light levels. Fast modulation of the beam up to $30 \mathrm{kHz}$ is implemented which allows background subtraction on the $100 \mu$ s time scale. The measurement covers the $0.9<\rho_{\text {pol }}<1.1$ range with $6-10 \mathrm{~mm}$ optical resolution at the measurement location which translates to 3-5 mm radial resolution at the midplane due to flux expansion. An automated routine has been developed which performs the background subtraction, the relative calibration, and the comprehensive error calculation, runs a Bayesian density reconstruction code, and loads results to the JET database. The paper demonstrates the capability of the APD system by analyzing fast phenomena like pellet injection and edge localized modes. https://doi.org/10.1063/1.4986621
\end{abstract}

\section{INTRODUCTION}

The Joint European Torus (JET) is the largest currently operating tokamak experiment. JET utilizes numerous plasma diagnostics in order to monitor a wide array of plasma parameters. One such diagnostic is the Lithium Beam Emission Spectroscopy (Li-BES) system, ${ }^{1}$ which is a routinely used diagnostic of magnetically confined plasmas.

The most common utilization of the Li-BES technique is to measure plasma edge density profiles, which feature has been demonstrated on numerous fusion devices: ASDEX Upgrade, ${ }^{2}$ COMPASS, ${ }^{3}$ DIII-D, ${ }^{4}$ EAST, ${ }^{5}$ JET, ${ }^{6}$ KSTAR, ${ }^{7}$ LHD,${ }^{8}$ TEXTOR, ${ }^{9}$ and W7-AS. ${ }^{10}$ Further capabilities such as measurement of the density fluctuations, ${ }^{2,11-13}$ poloidal flow velocity, ${ }^{14}$ ion temperature, ${ }^{15}$ impurity ion density profile, ${ }^{16}$ and edge current profile ${ }^{17}$ have been demonstrated. An alkaliBES system is being built at the W7-X stellarator and has been considered for JT-60SA. ${ }^{18}$ It has to be mentioned that the diagnostic can be extended to measure plasma edge current and potential fluctuations. ${ }^{19,20}$

On JET, besides the Li-BES diagnostic, several other methods are available for the measurement of the edge density profile. The high resolution Thomson scattering ${ }^{21}$

\footnotetext{
a) See the author list of "Overview of the JET results in support to ITER," by $\mathrm{X}$. Litaudon et al., to be published in Nuclear Fusion Special issue: Overview and summary reports from the 26th Fusion Energy Conference (Kyoto, Japan, 17-22 October 2016).
}

and the reflectometry ${ }^{22,23}$ are routinely used to determine plasma edge profiles. Compared to the Thomson scattering, Li-BES offers advantages in terms of temporal resolution while for reflectometry the knowledge of the measurement location is advantageous.

The measurement opportunities from the above list depend heavily on the signal to noise ratio (SNR), which in turn depends on the light yield and the noise sources of the system. The typical light yield at JET is in the $10^{8}$ photons/second range which translates to a maximum SNR of $\sim 10$ along the beam emission profile on the $250 \mathrm{kHz}$ bandwidth. This finally limits the JET Li-BES system to be capable of resolving plasma density profiles on the $100 \mu$ s time scale and to characterize scrape off layer (SOL) turbulence. Very strong MHD modes are resolvable in the confined region up to $50 \mathrm{kHz}$, but the power spectra are dominated by noise above this frequency. This paper recapitulates the recent upgrades of the system and describes the data evaluation methods which allowed us to improve the temporal resolution of the plasma edge density profile measurement from $10 \mathrm{~ms}^{6}$ to $100 \mu \mathrm{s}$.

The paper is organized as follows. An overview of the diagnostic's working principle and the hardware setup is shown in Sec. II, focusing on the critical elements for this paper. Section III describes the steps of the light profile calculation, namely, the problem of background correction (Sec. III A), the relative calibration (Sec. III B), and the error 
calculation (Sec. III C). Section IV introduces the density reconstruction method and its validation at JET. Two examples are shown in Sec. V to demonstrate the capabilities of the system, namely, a temporally resolved ELM (edge localized mode) event and a pellet injection.

\section{DIAGNOSTIC SETUP}

\section{A. BES working principle}

The working principle of such a diagnostic can be summarized as follows: ${ }^{24}$ an accelerated atomic beam is injected into the plasma, where the beam atoms are excited and ionized by plasma particles. The ionization process results in a gradual loss of the atoms in the beam. The ionization rate is such that the beam can penetrate only the edge of the plasma; thus, Li-BES systems are used for the electron density profile and fluctuation measurement of the outer plasma regions only, namely, the plasma edge and scrape off layer (SOL).

Spontaneous de-excitation of the beam atoms results in a characteristic photon emission at $670.8 \mathrm{~nm}$ that can be detected through an optical system. The distribution of the light emission along the beam (light profile) can be measured with a detector system, from which the electron density distribution (density profile) can be calculated. ${ }^{25,26}$ An important feature of such a diagnostic is that the beam attenuation sets the absolute magnitude of the plasma electron density during the density calculation; thus, no absolute but just relative intensity calibration of the light detection system channels is needed.

The Li-BES system consists of a lithium beam injector and an observation system.

\section{B. Lithium beam injector}

The lithium beam injector contains a thermionic ion source, an ion optics, two pairs of electrode plates for beam alignment and deflection, a sodium vapor neutralizer, and a flight tube. It is located on the top of the JET tokamak, shooting vertically down as illustrated on the left side in Fig. 1. The thermionic ion source emits ions when it is heated to $\sim 1300{ }^{\circ} \mathrm{C}$, and an extraction voltage is present. It is mounted in the Pierce electrode from which a two-stage ion optics extracts, accelerates, and focuses the lithium ions to $60 \mathrm{keV}$ energy. The beam can be aligned and chopped (deflected so as the beam does not enter the plasma) by applying a voltage on the deflection plates. The chopping is needed to be able to distinguish between the lithium emission and the background. The neutralizer is a sodium-filled oven which produces sodium vapor when it is heated to $\sim 250{ }^{\circ} \mathrm{C}$. Passing through the neutralizer, the lithium ions are neutralized by the charge exchange process. The lithium beam injector has an iron shield up to the neutralizer to keep the external magnetic field of the tokamak out of the region of the ion beam. The lithium atoms are injected into the plasma through the flight tube.

The JET lithium beam injector is a version of the original ASDEX lithium beam design ${ }^{27}$ and has been recently upgraded. The upgrade concerned the ion source ${ }^{28}$ and is capable of producing 1-2 mA ion equivalent neutral beam current in plasma. This upgrade also included the development of the beam deflection system which is now capable of chopping the beam up to $30 \mathrm{kHz}$. The details of such a deflection system design can be found in the study of Zoletnik et al. ${ }^{10}$

\section{Observation system}

The observation system can be divided into two parts as illustrated in the middle and on the right side in Fig. 1:

1. The periscope on the machine, inside the torus hall that images the beam emission on a fibre array through a rotatable mirror and an optical system. The beam is observed

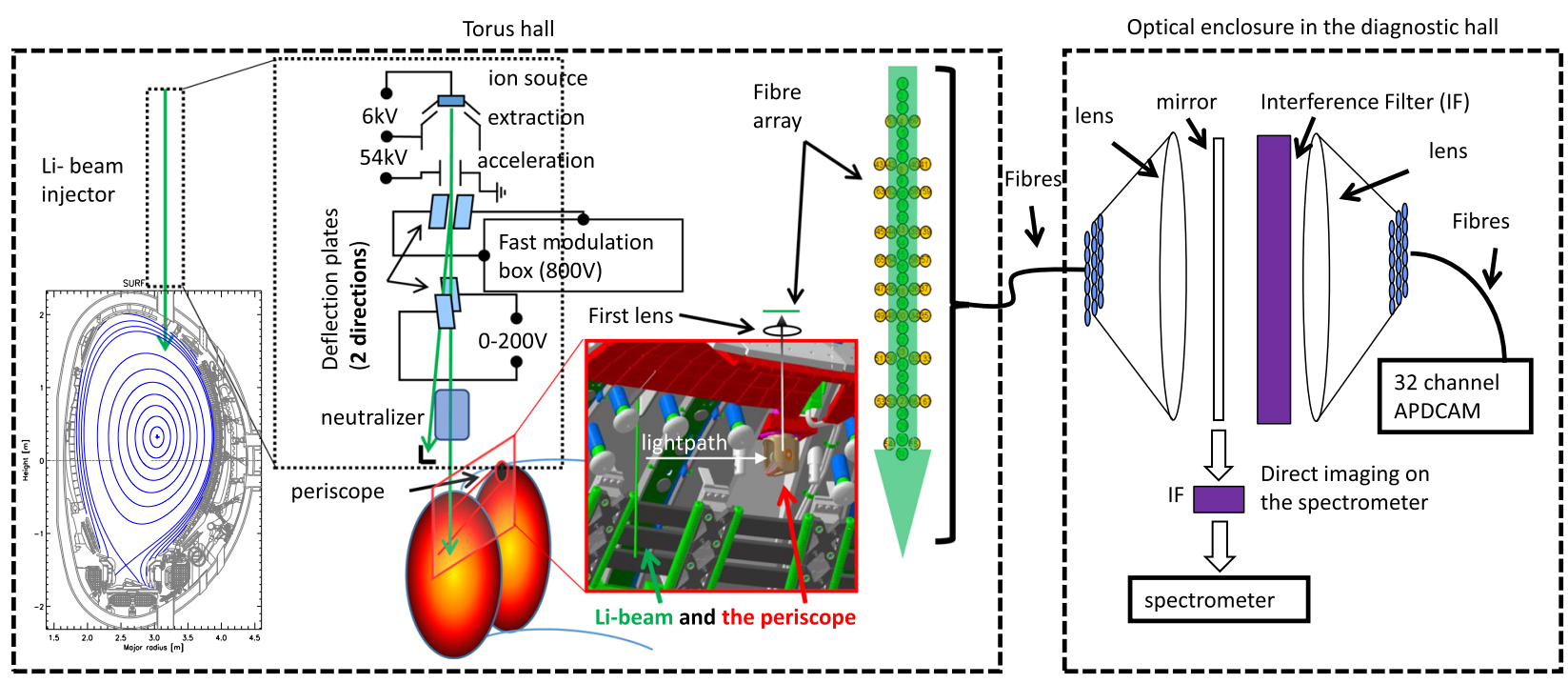

FIG. 1. JET Li-BES setup: the beam is injected from the top of the machine as indicated on the left. The block diagram shows the main parts of the injector: the heated ion source, the beam extractor and accelerator, the beam deflection system, and the neutralizer. Two poloidal cross sections of the plasma are illustrated by the red-yellow ellipses, showing the locations of the beam injection and the detection. The beam is observed through a periscope from a different toroidal cross section relative to the beam injection as indicated in the red box and the computer-aided design (CAD) drawing. The beam is imaged on a fibre array (green line) by an optical system in the periscope; the light path is illustrated with the white arrow. The image of the beam on the fibre array is illustrated by the large green arrow. The light is transmitted through optical fibres to an optical enclosure in the diagnostic hall. About $77 \%$ of the light is imaged on the 32 channel APDCAM through 2 large lenses, an interference filter, and a fibre array, while $11 \%$ on the spectrometer through a narrow mirror and another interference filter. 
from a quasi-tangential view looking downwards onto the beam at $0^{\circ}-45^{\circ}$ angle. The image diameter of the $1 \mathrm{~mm}$ diameter fibres on the beam is $6-10 \mathrm{~mm}$, depending on location, while the channel separation is defined by the spot size, since the optical fibres are touching each other in the fibre holder. This optical resolution translates to 3-5 $\mathrm{mm}$ radial resolution at the midplane due to flux expansion. The measurement range can be adjusted with an in-vessel mirror and covers typically the $0.9<\rho_{\text {pol }}<1.1$ range. In this paper, $\rho_{\text {pol }}$ refers to the normalized magnetic flux coordinate. The mirror is located in the far SOL; thus, it is from time to time subject to reflection loss due to deposited layers. This part can be replaced during a shutdown using the remote handling arm.

2. The other part is in an optical enclosure in the diagnostic hall; it processes the light transmitted by the $1 \mathrm{~mm}$ diameter optical fibres, 65 pieces altogether. This part has been upgraded as well in $2012^{6}$ and was equipped with a light splitting optical system that divides the light, coming from the torus hall through fibre optics, between a spectrometer and an avalanche photodiode (APD) camera (APDCAM, Fusion Instruments Kft). It was observed in the previous setup that even an upgraded spectrometer can process only about $10 \%$ of the light collected by the optics since the etendue of the optics exceeded that of the spectrometer. The spectrometer's etendue is defined by the slit width, which can be set according to the required spectral resolution, and the numerical aperture (acceptance angle), which is given. The new setup utilizes cylindrical lenses to narrow the image and match the numerical aperture and the slit width of the spectrometer, which is part of the light splitting optics. A 32 channel avalanche photodiode camera (APDCAM ${ }^{29}$ ) was installed to process the remaining part of the light. The APD branch of the observation system is equipped with an interference filter with FWHM $=2.4 \mathrm{~nm}$, which is needed due to the wide wavelength range of the Dopplershift of the beam emission. This wide interference filter cannot fully suppress the broadband radiation of the plasma which acts as a background to our measurement. The throughput of the system is as follows: $77 \%$ to the APDCAM, $11 \%$ to the spectrometer, and $12 \%$ loss, not considering the interference filters which have $\sim 75 \%$ peak transmittance.

\section{Detectors}

The spectrometer is capable of measuring the light emission from 26 fibres with $10 \mathrm{~ms}$ time resolution in a $10 \mathrm{~nm}$ spectral band. Spectral background correction, relative calibration of the channels after each plasma discharge by shooting into neutral gas, and several automated density reconstruction codes are implemented. The spectrometer provides spatial calibration as well since the position of the measurement along the beam can be calculated from the Doppler shift of the line emission and the observation geometry. This proved to be necessary due to the imprecision of the periscope mirror position setting caused by mechanical hysteresis and thermal expansion in the mirror actuator mechanism.
The APDCAM has 32 APD detectors out of which 26 measure the light from the same input fibres as the spectrometer, i.e., these channels are measured simultaneously by the two systems. The APDCAM system is optimized for the relatively low light intensities, set to $250 \mathrm{kHz}$ analog bandwidth, and measures with $500 \mathrm{kHz}$ sampling. The time resolution of the system is limited by the SNR which is in the range of 1-10 along the beam, when the ion gun is at its peak performance. This means that the relative error (statistical fluctuation of the light level) of the measurement can be reduced to an acceptable $5 \%$ level by $0.1-1 \mathrm{~ms}$ integration.

\section{LIGHT PROFILE CALCULATION}

The fast density deconvolution requires the calculation of a relatively calibrated beam light profile. In order to achieve this, the handling of the background light, the relative calibration of the channels, and the calculation of the errors are necessary. This section will show the steps of this analysis.

\section{A. Background correction}

The light measured by the observation system contains not only the beam light emission but also broadband radiation and multiple unresolved lines from low ionization state ions at the plasma edge as well, acting as the background in our measurement. The signal-to-background ratio of the system is in the range of 3-12. The background has to be subtracted from the signal to determine the beam light emission which is done by the modulation of the beam (chopping). The time period of the chopping determines the maximum time resolution of the system. The background correction becomes unreliable in the case of fast transient events which modulate the background on a time scale comparable to the chopping frequency.

Three chopping modes are available:

1. Slow modulation, when the beam is chopped out for each, e.g., 10th camera frame synchronized with the $10 \mathrm{~ms}$ long camera frame time.

2. Fast modulation, when the beam is continuously chopped with up to $30 \mathrm{kHz}$ frequency.

3. Mixed modulation, when the beam is on for every, e.g., 10th camera frame, off for the next frame to have sufficient background measurement for the spectrometer, and fast modulation in between.

The three chopping modes are illustrated in Fig. 2; note that the intervals with fast modulation are not to scale. The mixed modulation mode is illustrated with Fig. 3, where the APDCAM raw signal is shown in a time range that includes a camera synchronized $10 \mathrm{~ms}$ long full on and $10 \mathrm{~ms}$ long full off modulation along with the beginning of the $10 \mathrm{kHz}$ fast modulation in a zoomed subfigure. The background is calculated from each beam-off time interval, interpolated for the beam-on intervals, and subtracted from the signal level.

\section{B. Relative calibration}

The background corrected light intensity is proportional to the beam emission in the observed volume multiplied by the 
a)

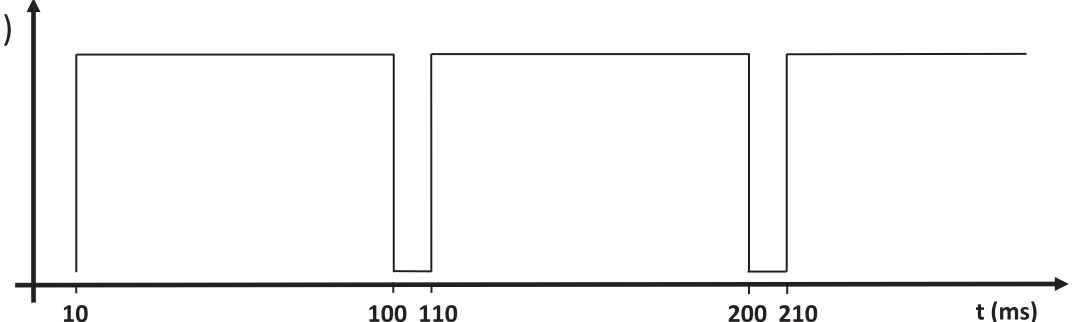

b)

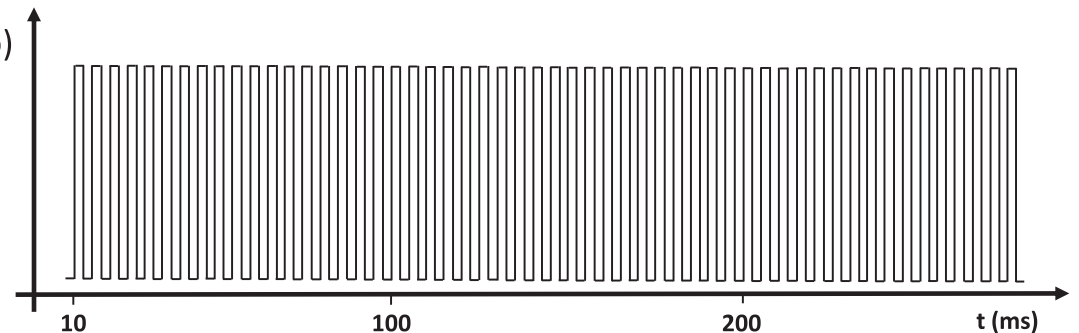

c)

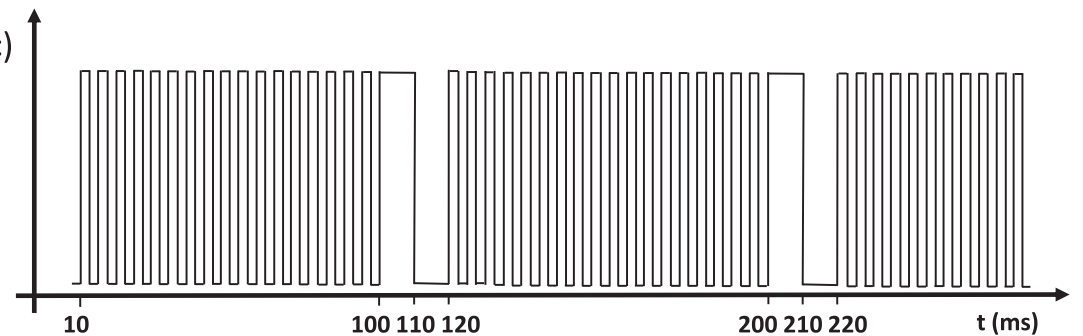

FIG. 2. Three beam modulation modes are illustrated: slow modulation (a), fast modulation (b), and mixed modulation (c) mode. The fast modulation period time is not to scale; it is in the $100 \mu \mathrm{s}$ range. solid angle of the observation, the transmission of the observation system (including the in-vessel optics, the fibres, the optical enclosure optics, and the interference filter), and the detection efficiency. The density profile calculation does not require the absolute value of the light intensity, as it was mentioned earlier, but just the relative light intensity distribution along the beam (light profile); thus, the relative calibration factors have to be determined. ${ }^{26}$ Two methods are implemented, as described below.

The first method calculates the calibration factors from a measurement in which the beam is injected into neutral

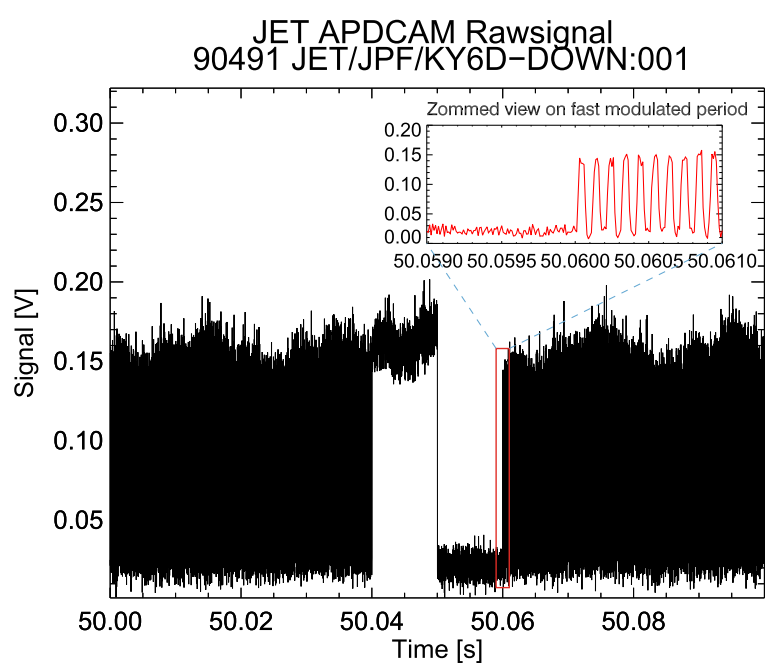

FIG. 3. Measured raw signal example in the mixed modulation mode. The main plot shows the raw signal including a slow, camera synchronized modulation interval, while the zoomed plot shows the beginning of the fast chopped period. gas where the beam emission can be considered homogeneous. In this case, the relative calibration factors are simply inversely proportional to the measured intensity. The spectrometer branch is calibrated after each plasma discharge with this method. The neutral gas pressure from the outgassing of the walls is sufficiently high (several times $10^{-5} \mathrm{mbar}$ ) to get a reasonable signal on the spectrometer with $0.5 \mathrm{~s}$ integration time. As the SNR of the APD branch in the gas measurement is low (0.1-1), this method can be used if the beam performs well, a sufficiently long $(\sim 3 \mathrm{~s})$ beam into gas shot takes place after the discharge, and the periscope mirror is clean.

Due to the limitations of the gas calibration, another method was implemented, based on the following considerations. The two branches of the detection system are measuring the same beam light emission at a fixed relative ratio and thus can be cross calibrated. Each APD signal is multiplied by a scaling factor to match the corresponding spectrometer signal. The scaling factor is calculated by matching the background corrected (spectral correction for the spectrometer, correction as described in Sec. III A for the APDCAM) light level in a plasma shot in the two systems for each channel, in a carefully chosen time interval, where no events are present that modulate the background too fast (i.e., faster than the $10 \mathrm{~ms}$ time scale of the spectrometer frame time). Typically a few $100 \mathrm{~ms}$ long L-mode part of a discharge is sufficient for this purpose.

\section{Error calculation}

In order to perform a density calculation from the light profile, errors should be quantified through a comprehensive error calculation, taking all sources into account, which are the following: 
- Random error

Electronic noise of the APD camera amplifiers Photon noise from plasma background Photon noise from lithium emission

- Systematic error

Gas calibration error

Cross calibration error

Steps of the calculation:

- Estimate the random error for each sample through signal-to-noise curve calculation.

- Calculate the lithium signal from the total and the background light and apply error propagation rules to estimate errors.

- Estimate the error of the gas calibration through modified standard error calculation.

- Estimate the error of the cross calibration taking errors of both detector systems into account.

The electronic noise and the photon noise are the random error sources in our measurement. The first step of the evaluation is a signal to noise curve calculation from the raw signal. The noise spectrum is measured at different light levels by illuminating the APD detectors with a light-emitting diode (LED) light source. This confirmed that the spectrum of both the electronic and the photon statistical noise is flat from about $1 \mathrm{kHz}$ up to about $150 \mathrm{kHz}$. The power in the $100-150 \mathrm{kHz}$ range of the signal spectra is considered purely electronic and photon noise as plasma and background light fluctuation amplitudes are below the noise level in the frequency range; thus, the total electronic and photon noise is calculated by the extrapolation of this power for the total $250 \mathrm{kHz}$ analog bandwidth. The photon noise has the Poisson statistics; thus, the variance (total power determined earlier) and the mean of the raw signal are proportional when there is light on the detector. The variance and the mean of the raw signal (containing all error sources) are calculated in each, e.g., $1 \mathrm{~ms}$ during the plasma discharge, and the resulting noise power versus signal level points are fitted with a linear function. This method is applied for each channel, and the resulting fits give the noise level for any signal level and channel in the examined plasma shot. This way we can calculate the random error level (square root of the variance) for each sample of the APDCAM.

During the background subtraction, the error propagation rules are taken into consideration. For a given beam-on interval, the background is the average of the mean light levels in the neighboring beam-off intervals. The total error of the background subtracted signal is the sum in quadrature of the signal and background errors. At this point, one has the noncalibrated lithium light level and the random error for each beam-on time.

The two types of relative calibrations described in Sec. III B bring the systematic error into the calculation. The error calculation of the APD gas calibration is complicated by offset drift and pickup noise that dominates white noise at low frequency. The $\sim 1 \mathrm{mV}$ signal level during a gas shot is small compared to the $\sim 10 \mathrm{mV}$ electronic noise. The photon statistical noise is negligible at this light level. The standard error of the mean value of multiple samples in the case of white noise decreases with the square root of the sample number, but a detailed analysis showed that the error decreases with the fourth root of the number of averaged samples above $\sim 2 \mathrm{~ms}$ integration time in our case. The effect is illustrated in Fig. 4 where the fluctuation power (variance) of an APD signal at different integration times is shown.

Taking this into consideration, the error of the gas calibration is calculated as follows. The APD data are recorded before, during and after the $\sim 3 \mathrm{~s}$ long beam injection into neutral gas, and the beam on and beam off phases are divided into $\mathrm{N}$ equidistant, $\sim 100 \mathrm{~ms}$ long parts each, and averaged within these intervals. $\mathrm{N}$ is chosen to have a fair statistics, 16 in our case. The error of the mean calibration factor in the full calibration time interval is then described by Eq. (1), where $\sigma\left(t_{\text {on }}\right)$ and $\sigma\left(t_{o f f}\right)$ are the standard deviation of the $\mathrm{N}$ element vector of beam intensities during beam on and beam off phases, respectively. The standard error calculated this way will be referred to as modified standard error (MSE),

$$
\operatorname{MSE}=\frac{\sqrt{\sigma^{2}\left(t_{o n}\right)+\sigma^{2}\left(t_{o f f}\right)}}{\sqrt[4]{N}} .
$$

The error of the cross calibration is calculated as follows. The calibrated lithium light level of a spectrometer channel is read from the JET database, while the APD data of the corresponding input fibre are averaged over each $10 \mathrm{~ms}$ long spectrometer frame time in the same time range. This way one has the time evolution of the lithium light and its error in both systems with identical sampling, and the time trace of the calibration factor can be achieved by dividing one vector by the other, applying error propagation rules. The calibration factor is the mean; the calibration factor error is the MSE of this time trace since the averaging goes over several $100 \mathrm{~ms}$ long dataset. The error of the spectrometer system calibration is the error of the spectral peak height during the beam into gas calibration. It was found to be negligible (less than $\sim 0.7 \%$ ) compared to other error sources, and thus it is not taken into account.

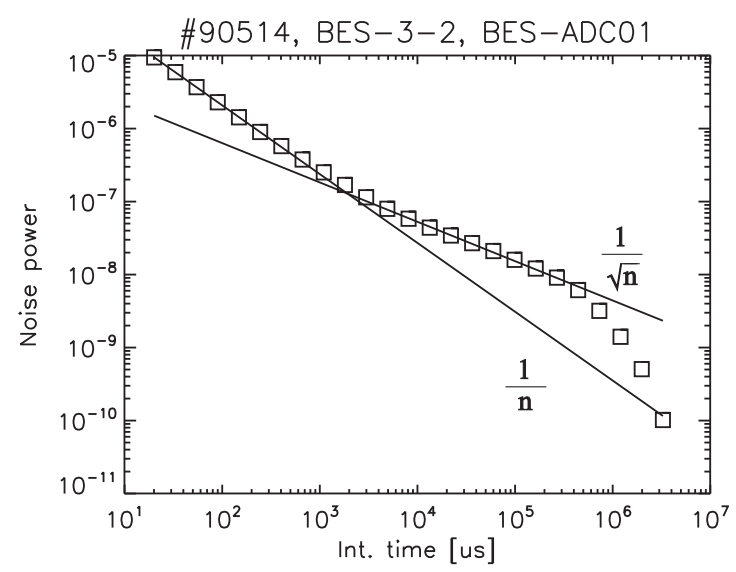

FIG. 4. The noise power in the signal (variance) as a function of integration time. Below $\sim 2 \mathrm{~ms}$ integration time, the noise is white and the fluctuation power is inversely proportional to the sample number. Above $\sim 2 \mathrm{~ms}$ integration time, the low frequency noise dominates the power, and the power falls only with the square root of the sample number. 


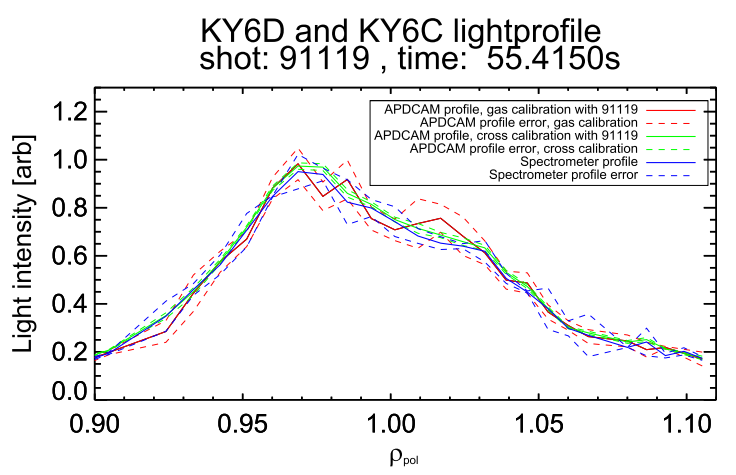

FIG. 5. JET light emission profile calculated from the gas calibrated (red line) and cross calibrated (green line) APDCAM data and from the spectrometer data (blue line). Dashed lines show the errors correspondingly.

After these considerations, one has both the random and systematic errors for each APD sample, which can be added up squared following the error propagation rules. In the case of temporal integration of the signal, the two errors are handled separately, and the systematic error is added only after the standard error of the mean has been calculated.

The results of the calibration methods and the error calculation can be seen in Fig. 5. The normalized light profiles calculated with gas and cross calibration are shown with red and green lines; the spectrometer profile is indicated with a blue line, while the dashed lines indicate the one-sigma errors of the profiles, respectively. The integration of the APDCAM profiles was set to the frame time of the spectrometer which is $10 \mathrm{~ms}$. It has to be emphasized that acceptable calibration precision for the APDCAM can be reached only with sufficiently high (several times $10^{-4} \mathrm{mbar}$ ) neutral gas pressure that can be reliably achieved by a dedicated gas calibration pulse. Yet no dedicated gas pulse is available at JET after the plasma shot; therefore, the gas calibration is carried out usually in the gas from the recombined plasma and the outgassing of the walls. The possibility of active gas injection after plasma termination is being investigated to increase the signal level during the gas calibration.

\section{DENSITY PROFILE RECONSTRUCTION}

A Bayesian density profile calculation method was implemented and validated against the numerical implementation of the method described in Ref. 26 on ASDEX Upgrade data. The code is written in Interactive Data Language (IDL) and utilizes the atomic physics core of the RENATE BES simulator. ${ }^{30,31}$

The atomic physics of the beam can be discussed within the collisional-radiative model. The light profile and the density profile are connected through the rate equations which is a coupled differential equation system. The integration is straightforward if one wants to calculate the light profile from a given density profile. One solution for the inverse problem is based on the Bayesian probability theory, that is, search the most probable density profile for a given light profile. The typical parametrization of the density profile for the free form Bayes calculation includes 13 spline parameters, plus the absolute calibration parameter and the initial lithium $2 p$ population parameter. Similar to Ref. 26, we describe the probability of a density profile as a product of the likelihood of the forward calculated light profile and two priors: one for the probability of the monotonicity and one for the curvature. For the likelihood of the forward calculated light profile, a Gaussian likelihood is assumed around the measured light profile with the error estimates as standard deviation. The priors reduce the unphysical density profiles. Weak monotonicity conditions are applied through the first prior, that is, a mostly monotonically increasing density profile toward the plasma center is assumed; however, if the data are not consistent with the monotonic profile, the code is capable of resolving the non-monotonic profile as well. The second prior penalizes the curvature of the density profile, that is, the reconstruction gives a smooth density profile. Both prior probability functions mainly act as weak constraints to the space of density profiles consistent with physical considerations. The advantage of this reconstruction method is that it can be applied to noisy data as in the case of the APDCAM measurement.

The error of the density profile is calculated by applying a local Gaussian density perturbation and increasing its amplitude so that $\tilde{\chi}^{2}$ of the forward calculated light profile increases by 1 . The locally perturbed density at this setting is considered as the $1 \sigma$ error at the given location. It has to be emphasized that the error calculated this way is not a local error since a local density perturbation influences the whole light profile after the perturbation through the atomic physics.

As a first validation step of the density calculation, we compared the results with density profiles calculated from spectrometer data. In Fig. 6, the blue line shows the density profile measured by the spectrometer, reconstructed by a nonstatistical, standard JET Li-BES reconstruction method. ${ }^{25}$ The red line shows the density profile measured by the APDCAM, reconstructed by the Bayesian method in the same time interval and time resolution (10 ms) for an L-mode case in Fig. 6(a) and for an H-mode case in Fig. 6(b).

As a second validation step, we compared the density profiles with other electron density measurements at JET. Figure 7 shows the electron density profile measured with four diagnostics: the lithium beam data calculated from the APDCAM are indicated with light blue triangles while those from the spectrometer are indicated with red triangles; the core Thomson scattering is shown with black plus signs while the high resolution Thomson scattering (HRTS) with dark blue diamonds, each mapped on the equatorial plane. The radial coordinate of the lithium beam was shifted $3 \mathrm{~cm}$ inwards to match the pedestal position of the HRTS; this is due to a known discrepancy of the magnetic equilibrium reconstruction algorithm that is being investigated. The last closed flux surface position has an uncertainity of $\pm 2.5 \mathrm{~cm}$ at the location of the Li-BES diagnostic. The chosen interval was an ELM-free H-mode period between 55.5 and $56 \mathrm{~s}$ in shot \#91119. The time resolution of the APDCAM reconstruction was set to $10 \mathrm{~ms}$ to match that of the spectrometer. The profiles show fairly good agreement up to the pedestal top $\left(\rho_{p o l} \simeq 0.95\right)$, while Li-BES loses sensitivity thereafter due to ionization, and the reconstruction stops. 
KY6D and KY6C density profile, shot: 91119
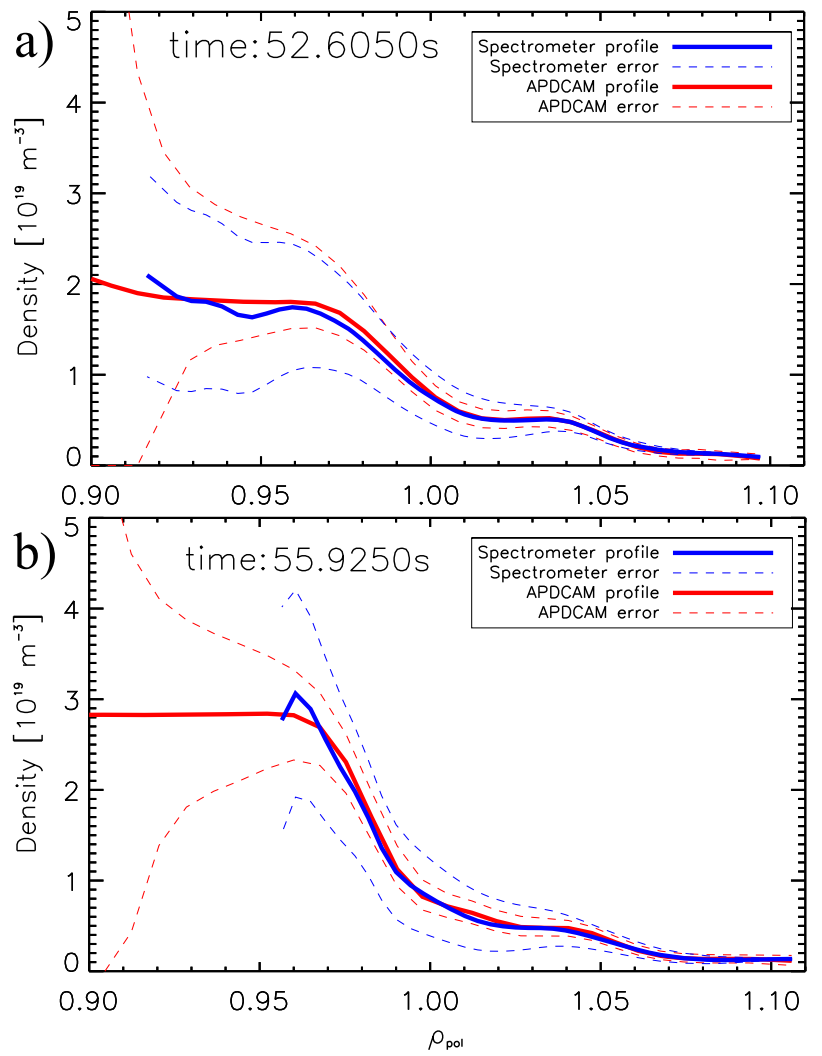

FIG. 6. JET density profile calculated from the spectrometer data (blue line) and from APDCAM data (red line) for (a) L-mode and (b) H-mode cases. Dashed lines show the respective errors.

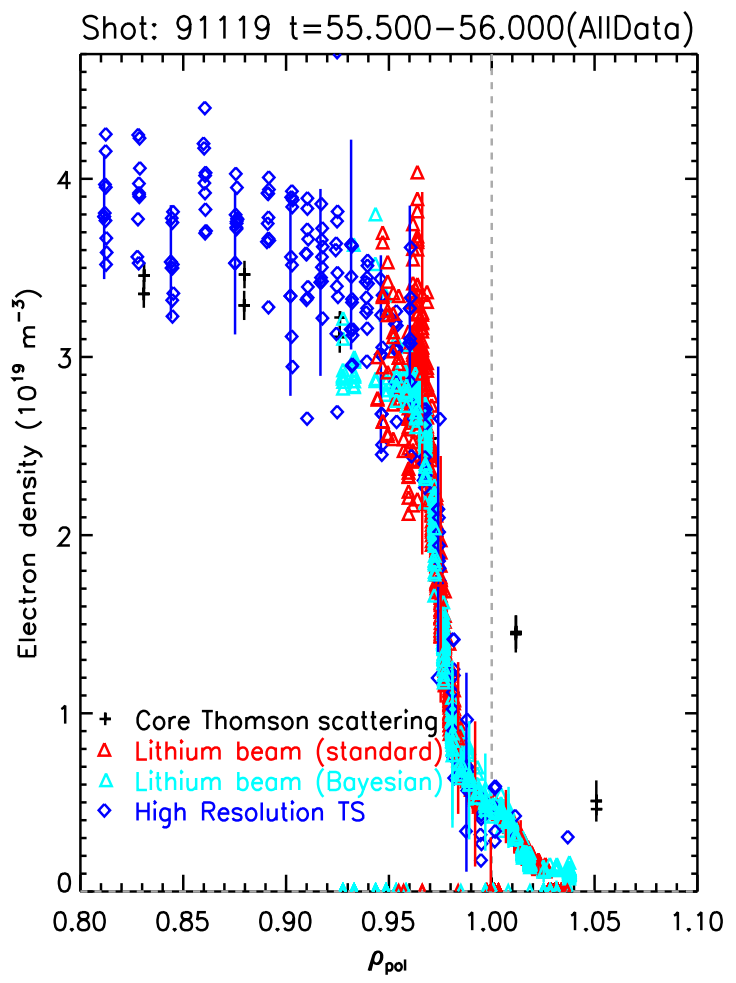

FIG. 7. JET density profile calculated from the APD data with our Bayesian code (light blue), from spectrometer data with a deterministic code (red), core Thomson scattering diagnostic (black), and high resolution Thomson scattering diagnostic (dark blue).

\section{DENSITY PROFILE MEASUREMENT EXAMPLES}

The temporal resolution and spatial resolution of the JET Li-BES diagnostic are to be demonstrated in this section. Two temporally resolved fast events, an ELM crash and a pellet injection case, are presented in this section.

\section{A. ELM cycle}

Edge Localized Modes (ELMs) are fast, periodic plasma events in the H-mode, which are of interest due to their role played in impurity transport and the high particle and heat load carried by them that is hazardous in terms of machine operation and safety. ${ }^{32}$ Figure 8(a) shows the time evolution of the density profile determined with the method presented earlier during an ELM event as a contour plot. The $\mathrm{x}$ axis is time (s), and the $y$ axis is the height above the mid-plane coordinate $(\mathrm{m})$ along the beam that propagates downwards. The plot shows that the pedestal crashes and the density profile flattens due to the ELM. The increased SOL density after the crash can correspond both to outward particle flux and increased recycling flux of neutrals being ionized by the SOL plasma. The last closed flux surface (LCFS) is marked with a white dashed horizontal line, while the colored vertical lines correspond to the time slices indicated in Fig. 8(c). The inner divertor beryllium signal is shown as a reference in Fig. 8(b), where the increased load due to the ELM event is immediate on the density measurement time scale. The density profiles during the
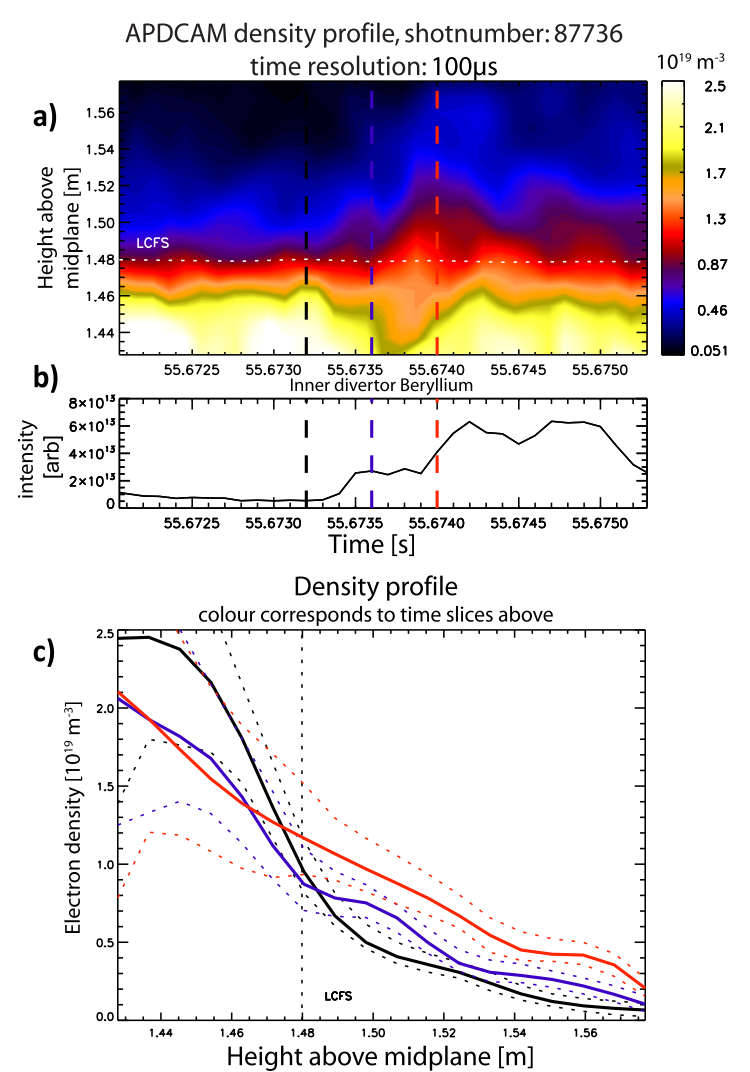

FIG. 8. Temporally resolved ELM crash: (a) contour plot of the density profile time evolution and the selected time slices for subplot (c), (b) inner divertor beryllium signal, (c) density profile at the selected time slices (solid line) and the corresponding errors (dotted line). The color of the lines corresponds to the selected times in subplot (a) and (b), temporal evolution order: black-blue-red. 
ELM crash (solid line) and the corresponding errors (dotted line) are shown in Fig. 8(c). The color of the profiles corresponds to the vertical dashed lines in Figs. 8(a) and 8(b) showing the time of the selected profile; thus, the time evolution order is black-blue-red. The black profile is taken from before, while the blue and the red profiles are taken during the ELM crash. First, the pedestal top density is decreased by $20 \%$, while the density in the SOL is increased. It is worthwhile to point out that the error of the density profile at a given radial position is increased by a factor two during the ELM due to the increased background light.

\section{B. Pellet injection}

Pellet injection is recognized as an important tool for plasma fuelling and ELM control. To understand the underlying processes of the pellet ablation and particle deposition, it is of interest to detect the pellet-caused density increase in the time scale of a few tens of microseconds. Pellets can trigger ELMs in H-mode plasmas therefore to disentangle the effect of fuelling and density change caused by the triggered ELMs; the dynamics of the pellet-caused density profile change was investigated in L-mode discharges. The injection of the cryogenic deuterium pellet was carried out with the low field side pellet injector. As a typical example, Fig. 9(a) shows the

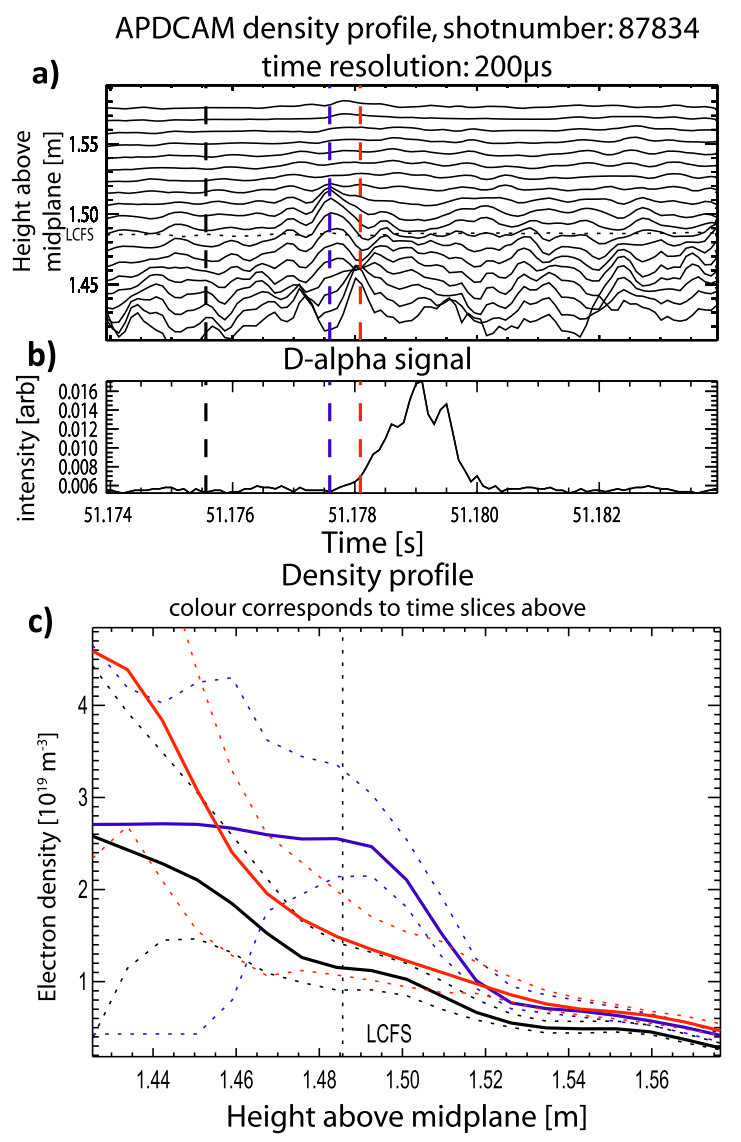

FIG. 9. Temporally resolved pellet penetration: (a) level plot of the density profile time evolution, (b) divertor D-alpha signal below, (c) density profile at the selected time slices (solid line), and the corresponding errors (dotted line), temporal evolution order: black-blue-red. Level plot interpretation: each line represents the time trace of the density relative to the average density over the whole interval at a fixed radial position. density change relative to the average density over the investigated interval at different radial locations. The pellet ablation monitor (D-alpha light) is also plotted in Fig. 9(b) as a reference. The pellet-induced density increase propagating into the plasma is clearly seen in Fig. 9(a). The peak is propagating with about $100 \mathrm{~m} / \mathrm{s}$ speed $(5 \mathrm{~cm} / 500 \mu \mathrm{s})$ that is in the order of the typical pellet speed. Figure 9(c) shows the density profiles (solid line) and the corresponding errors (dotted line) at the time slices indicated in Figs. 9(a) and 9(b), temporal evolution order: black-blue-red. The black profile is the L-mode density profile before the pellet injection; the blue profile is taken at the time when the pellet reached the SOL, while the red profile corresponds to the time when the pellet already reached the confined region. The effect of the electron temperature change was investigated and was found to be negligible even in this case, when the edge temperature decreases by a factor 4 (from $200 \mathrm{eV}$ to $50 \mathrm{eV}$ ) during the fast event.

\section{SUMMARY AND CONCLUSIONS}

The Li-BES system at the JET tokamak is a routinely used diagnostic for electron density profile measurement. The device has been upgraded in the recent years, and a fast beam emission light detection system was implemented. The fast background correction through chopping of the beam and the simultaneous measurement of the beam emission light with the APDCAM and the spectrometer system enable us to produce $\sim 1 \mathrm{~cm}$ spatial and $\sim 100 \mu$ s temporal resolution SOL and plasma edge density profiles up to the pedestal top. The temporal resolution, compared to prior capabilities, is improved by a factor of 100 .

Comparing to a recent upgrade of the ASDEX Upgrade Li-beam system, ${ }^{2,33}$ one can observe that the light intensity in the fast branch is similar; therefore, the time resolution of the two diagnostics is also similar. The distinct feature of the JET Li-beam system is the faster chopper which enables precise background measurement on a $100 \mu$ s time scale.

On the other hand, the SNR of the JET Li-beam diagnostic does not allow measurement on a time scale below $100 \mu \mathrm{s}$, thus zonal flow and edge turbulence measurements like on TEXTOR $^{14}$ are still not possible. Determining SOL and edge turbulence spectra and correlation functions like on ASDEX Upgrade $^{2}$ and Wendelstein $7-\mathrm{AS}^{11}$ are possible and will be reported in a separate paper.

This feature of the system enables us to resolve the evolution of fast transient events, such as ELM-s, pellet injection, $\mathrm{L}-\mathrm{H}$ transition, and $\mathrm{M}$-mode, ${ }^{34}$ which are in the focus of interest at magnetically confined devices.

\section{ACKNOWLEDGMENTS}

This work has been carried out within the framework of the EUROfusion Consortium and has received funding from the Euratom research and training programme 2014-2018 under Grant Agreement No. 633053. The views and opinions expressed herein do not necessarily reflect those of the European Commission. 
${ }^{1}$ M. Brix, A. Korotkov, M. Lehnen, P. Morgan, K. McCormick, J. Schweinzer, D. Summers, and J. Vince, in Proceedings of 28th EPS Conference on Controlled Fusion and Plasma Physics (ECA-European Conference Abstracts, Madeira, 2001), Vol. 25, p. 389.

${ }^{2}$ M. Willensdorfer, G. Birkenmeier, R. Fischer, F. M. Laggner, E. Wolfrum, G. Veres, F. Aumayr, D. Carralero, L. Guimarais, B. Kurzan, and ASDEX Upgrade Team, Plasma Phys. Controlled Fusion 56, 025008 (2014).

${ }^{3}$ G. Anda, A. Bencze, M. Berta, D. Dunai, P. Hacek, J. Krbec, D. Réfy, T. Krizsanóczi, S. Bató, T. Ilkei, I. Kiss, G. Veres, and S. Zoletnik, Fusion Eng. Des. 108, 1 (2016).

${ }^{4}$ H. Stoschus, D. M. Thomas, B. Hudson, J. M. M. Burgos, and J. Schweinzer, Rev. Sci. Instrum. 83, 10D508 (2012).

${ }^{5}$ G. Anda, D. Dunai, M. Lampert, T. Krizsanóczi, J. Németh, S. Bató, Y. U. Nam, G. H. Hu, and S. Zoletnik, Rev. Sci. Instrum. 89, 013503 (2018).

${ }^{6}$ M. Brix, D. Dodt, D. Dunai, I. Lupelli, S. Marsen, T. Melson, B. Meszaros, P. Morgan, G. Petravich, D. Réfy et al., Rev. Sci. Instrum. 83, 10 D533 (2012).

${ }^{7}$ M. Lampert, G. Anda, A. Czopf, G. Erdei, D. Guszejnov, Á. Kovácsik, G. I. Pokol, D. Réfy, Y. U. Nam, and S. Zoletnik, Rev. Sci. Instrum. 86, 073501 (2015).

${ }^{8}$ T. Morisaki, A. Komori, O. Motojima, and LHD Experimental Group, Rev. Sci. Instrum. 74, 1865 (2003).

${ }^{9}$ D. Dunai, S. Zoletnik, J. Sárközi, and A. R. Field, Rev. Sci. Instrum. 81, 103503 (2010).

${ }^{10}$ S. Zoletnik, G. Petravich, A. Bencze, M. Berta, S. Fiedler, K. McCormick, and J. Schweinzer, Rev. Sci. Instrum. 76, 073504 (2005).

${ }^{11}$ S. Zoletnik, M. Anton, M. Endler, S. Fiedler, M. Hirsch, K. McCormick, J. Schweinzer, and W7-AS Team, Phys. Plasmas 6, 4239 (1999).

${ }^{12}$ D. M. Thomas, A. W. Hyatt, and M. P. Thomas, Rev. Sci. Instrum. 61, 3040 (1990).

${ }^{13}$ Y. U. Nam, S. Zoletnik, M. Lampert, and Á. Kovácsik, Rev. Sci. Instrum. 83, 10D531 (2012).

${ }^{14}$ S. Zoletnik, L. Bardoczi, A. Kraemer-Flecken, Y. Xu, I. Shesterikov, S. Soldatov, G. Anda, D. Dunai, G. Petravich, and TEXTOR Team, Plasma Phys. Controlled Fusion 54, 065007 (2012).

${ }^{15}$ M. Reich, E. Wolfrum, J. Schweinzer, H. Ehmler, L. D. Horton, J. Neuhauser, and ASDEX Upgrade Team, Plasma Phys. Controlled Fusion 46, 797 (2004).

${ }^{16}$ E. Wolfrum, J. Schweinzer, M. Reich, L. D. Horton, and C. F. Maggi, Rev. Sci. Instrum. 77, 033507 (2006).

${ }^{17}$ K. Kamiya, T. Fujita, A. Kojima, and H. Kubo, Rev. Sci. Instrum. 81, 033502 (2010).

${ }^{18}$ Ö. Asztalos, G. Pokol, D. Dunai, G. Boguszlavszkij, Á. Kovácsik, M. Hellermann, K. Kamiya, T. Suzuki, and A. Kojima, Fusion Eng. Des. 123, 861 (2017).
${ }^{19}$ M. Berta, G. Anda, M. Aradi, A. Bencze, C. Buday, I. Kiss, S. Tulipán, G. Veres, S. Zoletnik, J. Havlícek, and P. Háček, Fusion Eng. Des. 88, 2875 (2013).

${ }^{20}$ J. Galdon-Quiroga, J. Rivero-Rodriguez, M. Garcia-Munoz, G. Birkenmeier, E. Viezzer, J. Ayllon-Guerola, M. Dunne, J. GarciaLopez, J. Gonzalez-Martin, M. Jimenez-Ramos, M. Rodriguez-Ramos, L. Sanchis-Sanchez, E. Wolfrum, and ASDEX Upgrade Team, J. Instrum. 12, C08023 (2017).

${ }^{21}$ R. Pasqualotto, P. Nielsen, C. Gowers, M. Beurskens, M. Kempenaars, T. Carlstrom, D. Johnson, and JET-EFDA Contributors, Rev. Sci. Instrum. 75, 3891 (2004).

${ }^{22}$ L. Meneses, L. Cupido, A. A. Ferreira, S. Hacquin, M. Manso, and JET-EFDA Contributors, Rev. Sci. Instrum. 77, 10 E927 (2006).

${ }^{23}$ G. Wang, E. J. Doyle, W. A. Peebles, L. Zeng, T. L. Rhodes, S. Kubota, X. Nguyen, and N. A. Crocker, Rev. Sci. Instrum. 75, 3800 (2004).

${ }^{24}$ E. Wolfrum, F. Aumayr, D. Wutte, H. Winter, E. Hintz, D. Rusbüldt, and R. P. Schorn, Rev. Sci. Instrum. 64, 2285 (1993).

${ }^{25}$ J. Schweinzer, E. Wolfrum, F. Aumayr, M. Pockl, H. Winter, R. P. Schorn, E. Hintz, and A. Unterreiter, Plasma Phys. Controlled Fusion 34, 1173 (1992).

${ }^{26}$ R. Fischer, E. Wolfrum, J. Schweinzer, and ASDEX Upgrade Team, Plasma Phys. Controlled Fusion 50, 085009 (2008).

${ }^{27}$ K. McCormick, S. Fiedler, G. Kocsis, J. Schweinzer, and S. Zoletnik, Fusion Eng. Des. 34-35, 125 (1997).

${ }^{28}$ G. Bodnar, S. Bató, S. Zoletnik, M. Brix, and R. Gomes, JET Internal Report EFDA-JET-CP(14)05/11, 2014.

${ }^{29}$ A. R. Field, D. Dunai, R. Gaffka, Y.-C. Ghim, I. Kiss, B. Meszaros, T. Krizsanoczi, S. Shibaev, and S. Zoletnik, Rev. Sci. Instrum. 83, 013508 (2012).

${ }^{30}$ D. Guszejnov, G. I. Pokol, I. Pusztai, D. Réfy, S. Zoletnik, M. Lampert, and Y. U. Nam, Rev. Sci. Instrum. 83, 113501 (2012).

${ }^{31}$ I. Pusztai, G. Pokol, D. Dunai, D. Réfy, G. Por, G. Anda, S. Zoletnik, and J. Schweinzer, Rev. Sci. Instrum. 80, 083502 (2009).

${ }^{32}$ H. Zohm, Plasma Phys. Controlled Fusion 38, 105 (1996).

${ }^{33}$ M. Willensdorfer, E. Wolfrum, R. Fischer, J. Schweinzer, M. Sertoli, B. Sieglin, G. Veres, F. Aumayr, and ASDEX Upgrade Team, Rev. Sci. Instrum. 83, 023501 (2012).

${ }^{34}$ E. R. Solano, N. Vianello, E. Delabie, J. Hillesheim, P. Buratti, D. Réfy, I. Balboa, A. Boboc, R. Coelho, B. Sieglin, S. Silburn, P. Drewelow, S. Devaux, D. Dodt, A. Figueiredo, L. Frassinetti, S. Marsen, L. Meneses, C. Maggi, J. Morris, S. Gerasimov, M. Baruzzo, M. Stamp, D. Grist, I. Nunes, F. Rimini, S. Schmuck, I. Lupelli, C. Silva, and JET Contributors, Nucl. Fusion 57, 022021 (2017). 